\title{
Functional Mediastinal Paraganglioma Irrigated by Coronary Artery Collaterals Associated With Bilateral Renal Artery Fibro-Dysplasia in a Hypertensive Patient
}

\author{
Khaled Elenizi ${ }^{\mathrm{a}, \mathrm{b}, \mathrm{d}}$, Rasha Alharthi ${ }^{\mathrm{b}}$, Anthony Matta ${ }^{\mathrm{b}, \mathrm{c}}$, Michel Galinier ${ }^{\mathrm{b}}$
}

\begin{abstract}
We present a symptomatic functional anterior mediastinal paraganglioma (PGL) in a 56-year-old woman. The patient was known to have renovascular resistant hypertension caused by fibromuscular dysplasia diagnosed several years ago. The diagnosis of PGL was confirmed by 123I-metaiodobenzylguanidine scintigraphy, $18 \mathrm{~F}$ FDOPA positron emission tomography/computed tomography and a thoracic tomographic scan which revealed an anterior mediastinal tumor. Complete tumor resection was done through a sternotomy and extracorporeal circulation with cardiac arrest. The histological examination of the operative specimen was characteristic of PGL.
\end{abstract}

Keywords: Mediastinal paragangliomas; Renal artery stenosis; Fibromuscular dysplasia; Coronary vessel irrigation

\section{Introduction}

Functional paragangliomas (PGLs) are non-epithelial neuroendocrine neoplasms, derived from paraganglia of the sympathetic and parasympathetic nervous systems [1]. They can be found in all normal anatomical sites of paraganglia where chromaffin tissue is located (para-aortic sympathetic chain, sympathetic chain in the neck and mediastinum, within organs of Zuckerkandl at the origin of the inferior mesenteric artery and the wall of the urinary bladder). It can arise from all locations in the body except within the brain and in bone [2]. PGLs can be classified as functional or non-functional PGLs based on the ability of synthesizing and releasing catecholamines. PGLs that form in the adrenal glands are called pheochromocytomas (PCCs) and those that form outside the adrenal glands

Manuscript submitted September 25, 2019, accepted October 7, 2019

aDepartment of Internal Medicine, College of Medicine, Prince Sattam bin Abdulaziz University, Alkharj 11942, Saudi Arabia

${ }^{\mathrm{b}}$ Cardiovascular Department, Rangueil Hospital, Toulouse, France

cFaculty of Medicine, Holy Spirit University of Kaslik, Kaslik, Lebanon

${ }^{\mathrm{d} C}$ Corresponding Author: Khaled Elenizi, Cardiovascular Department, Rangue-

il Hospital, Toulouse, France. Email: kelenizi@yahoo.com

doi: https://doi.org/10.14740/jmc3379 are called extra-adrenal PGLs. They are located in the adrenal medulla in $90 \%$ of cases and in the mediastinum in $<2 \%$ [3]. PGLs are generally slow-growth tumors and rarely develop into malignant ones [4].

The coexistence of renal artery stenosis (RAS) and PCC/ PGL is rare. However, it has been recognized in 1958 by Harrison et al [5] in a 16-year-old girl. The largest study in this subject so far found that among 269 patients with PCC, only eight patients have a coexisting RAS [6]. The mechanisms of RAS were variant: extrinsic compression in three patients, intimal fibroplasia in two, possible catecholamine-induced vasoconstriction in two, iatrogenic in one and atherosclerosis in one. In addition, a more recent retrospective study [7] on Indian population included patients with extra-adrenal PGL and found that RAS is often associated with extra-adrenal PGL (prevalence of 30\%) in patients with PGL, supporting that probably norepinephrine secreted abundantly by the extra-adrenal PGLs is potentially more potent vasoconstrictors in comparison to those secreted by PCC.

In reviewing the literature, we found only a few cases that describe similar association. However, the clinical implication of this coexistence and its mechanism is not well established. The most common mechanism involved in RAS is extrinsic compression mainly in PCC patients and can be corrected surgically. Another mechanism is the development of fibromuscular dysplasia like morphology; this mechanism is not proved yet. It is hypothesized that elevated local catecholamines levels initiate a sustained transient arterial spasm also called "pseudostenosis", that cumulate giving dysplasia-like morphology changes [8]. Such stenosis becomes significant during a hypertensive PCC/PGL crisis and can be triggered by aortography [9]. This stenotic variability mediated by the released catecholamines makes the diagnosis of renovascular hypertension challenging and could be reversible by alpha blockers or after excising the tumor $[9,10]$. For that, undergoing renal revascularization or balloon angioplasty in such patients before a diagnostic trial of alpha blockers or excising the tumor is not appropriate. The understanding of this entity is crucial for providing the appropriate management.

\section{Case Report}

A 56-year-old female known case of nonfunctional bilateral adrenal adenomas, fibromyalgia, hypercholesteremia treated with statin, ulcerative colitis treated with mesalamine and 


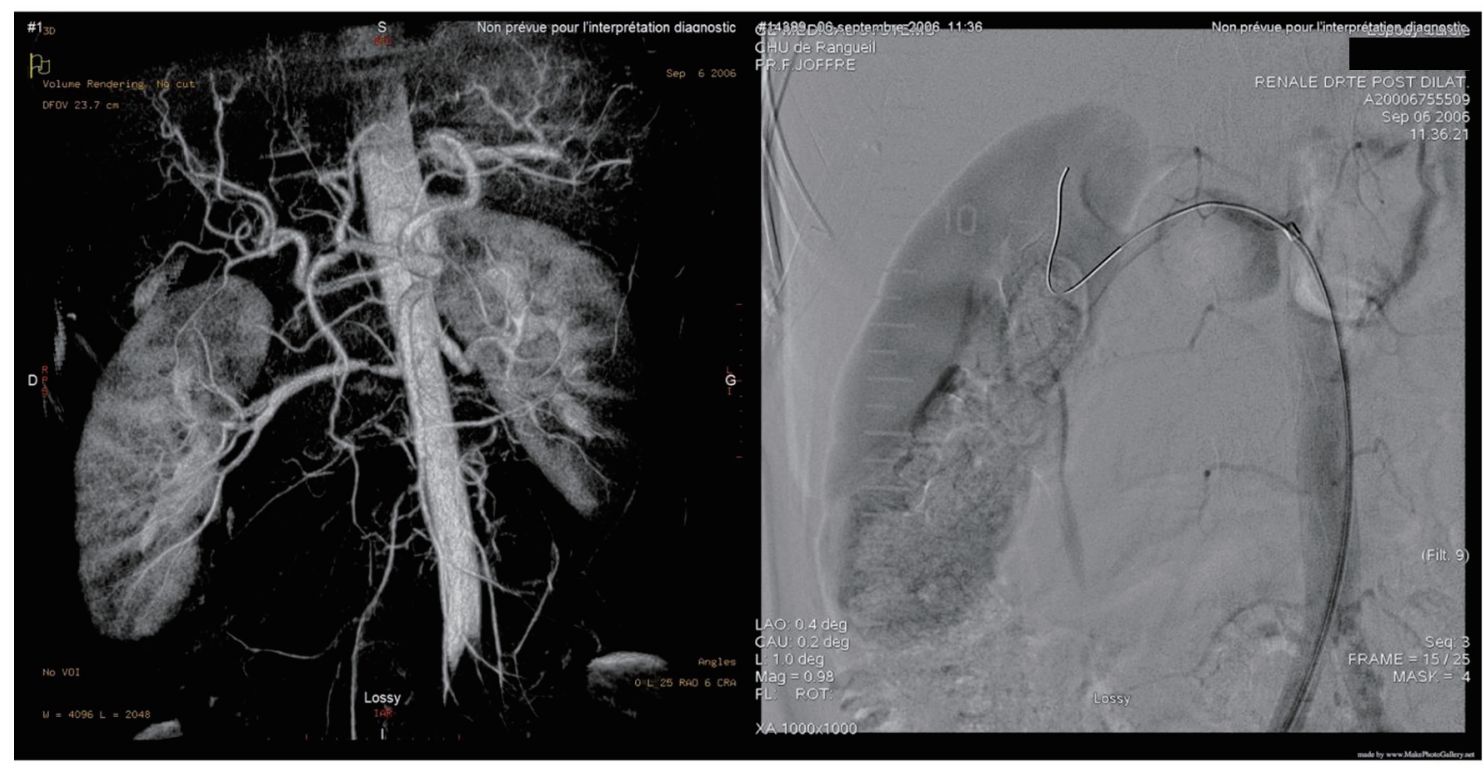

Figure 1. Balloon angioplasty w/o stenting.

parathyroidectomy for primary hyperparathyroidism. Her history started at 34 years of age when she was found to have severe symptomatic hypertension resistant to treatment. Upon evaluation of secondary causes, a unilateral severe left RAS caused by fibromuscular dysplasia was identified and treated with balloon angioplasty without stenting. Nine years later, she presented with another episode of severe hypertension unveiling a severe right RAS that was treated similarly with balloon angioplasty (Fig. 1). Figure 2 shows normal chest X-ray. The patient continued taking antihypertensive medications which included valsartan/hydrochlorothiazide $80 / 12.5 \mathrm{mg}$ and bisoprolol $10 \mathrm{mg}$ once daily.

At a follow-up visit, the patient was found to have resistant diastolic hypertension on ambulatory blood pressure monitoring and complained mainly of generalized fatigue. Lab tests showed normal blood electrolytes and normal kidney function. Upon evaluation, urinary catecholamines were found to be elevated: normetanephrine $910 \mu \mathrm{g} / 24 \mathrm{~h}$ (normal < $600 \mu \mathrm{g} / 24 \mathrm{~h}$ ), metanephrines were normal and noradrenaline was elevated at $229 \mu \mathrm{g} / 24 \mathrm{~h}$ (normal < $105 \mu \mathrm{g} / 24 \mathrm{~h})$. Plasmatic metanephrines were also elevated. Renal artery echo Doppler found a bilateral non-significant RAS which was not seen on a contrastenhanced CT scan (Fig. 3), meanwhile showing a mediastinal mass located between the pulmonary artery and the left atrium measuring $43 \times 23 \times 28 \mathrm{~mm}$ and invading the adjacent structures (Fig. 4). Searching for the source of high catecholamine levels, MIBG scintigraphy was done showing mediastinal fixation suggestive of PGL (Fig. 5). The result was confirmed by $18 \mathrm{~F}-F D O P A$ PET scan which found an intense mediastinal fixation compatible with PGL (Figs. 6 and 7). It also showed two asymmetric limited fixations at the level of the suprarenal glands. Transthoracic echography (TTE) showed a normal cardiac function with unusual hyperechogenic mass adjacent to the pulmonary artery (Fig. 8). As part of the preoperative evaluation, the patient underwent coronary angiography by trans-radial approach that surprisingly found a vascularization of the mediastinal mass by two collaterals originating from the proximal and distal part of circumflex artery (Fig. 9).

Finally, a total surgical resection of the tumor under general anesthesia was done (Fig. 10) and the patient was treated with urapidil and bisoprolol weeks before the operation. There were no complications during the operation. The histopathology of the specimen confirms the diagnosis of PGL (Fig. 11).

\section{Discussion}

Interestingly, our patient presented with renovascular hyper-

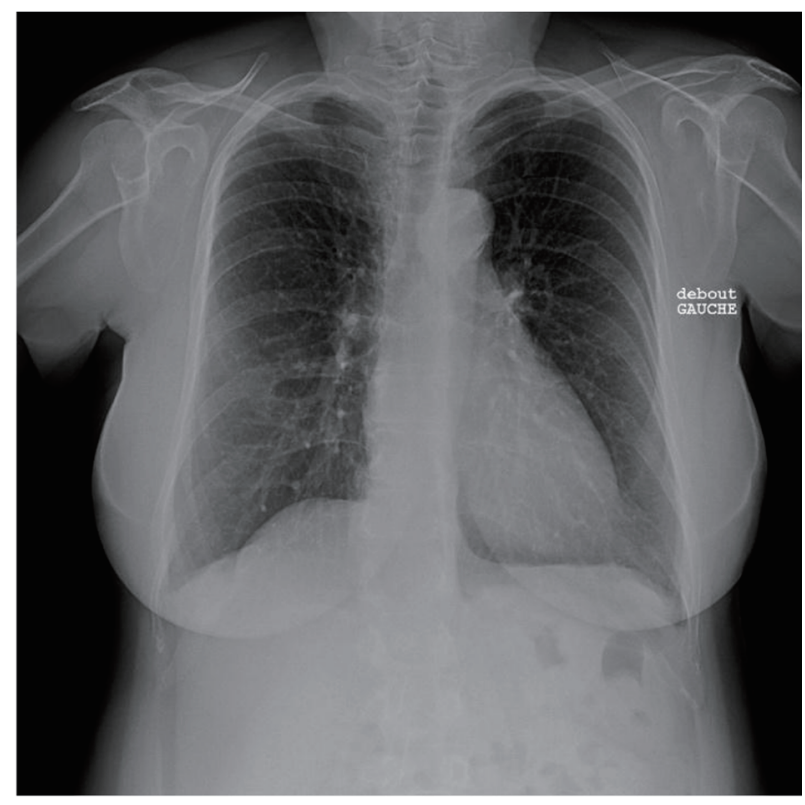

Figure 2. Normal chest X-ray. 


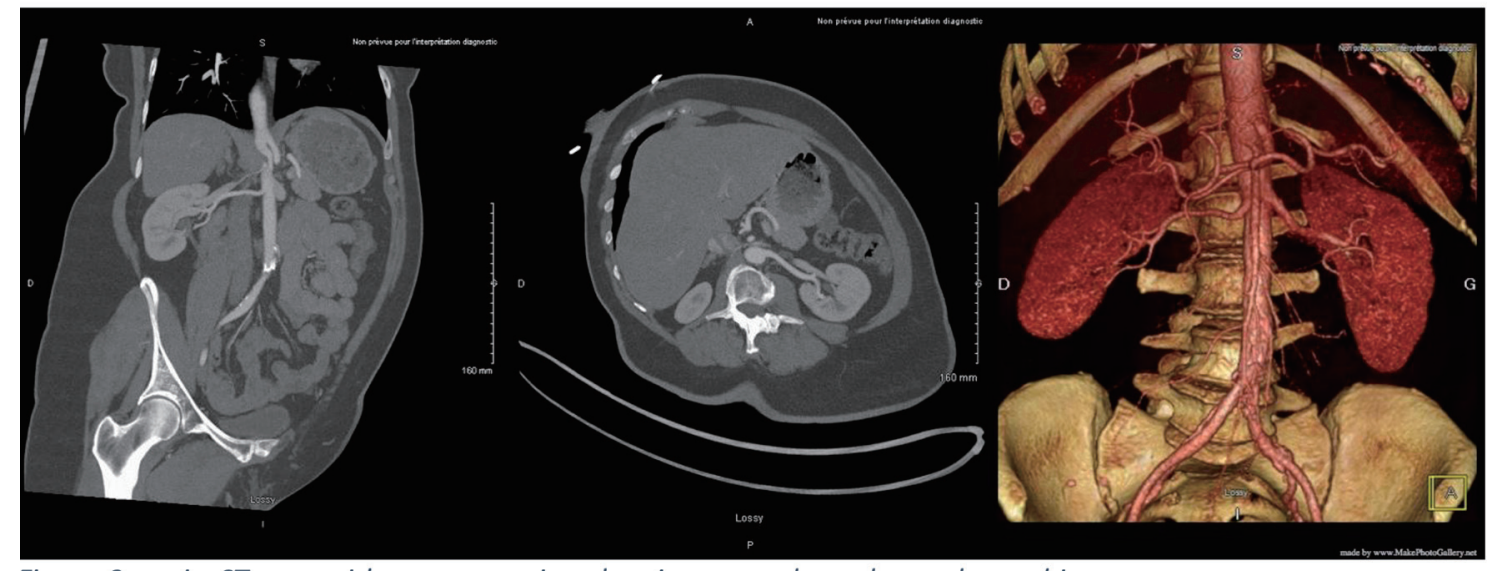

Figure 3. Angio-CT scan with reconstruction showing normal renal vascular architecture. CT: computed tomography.

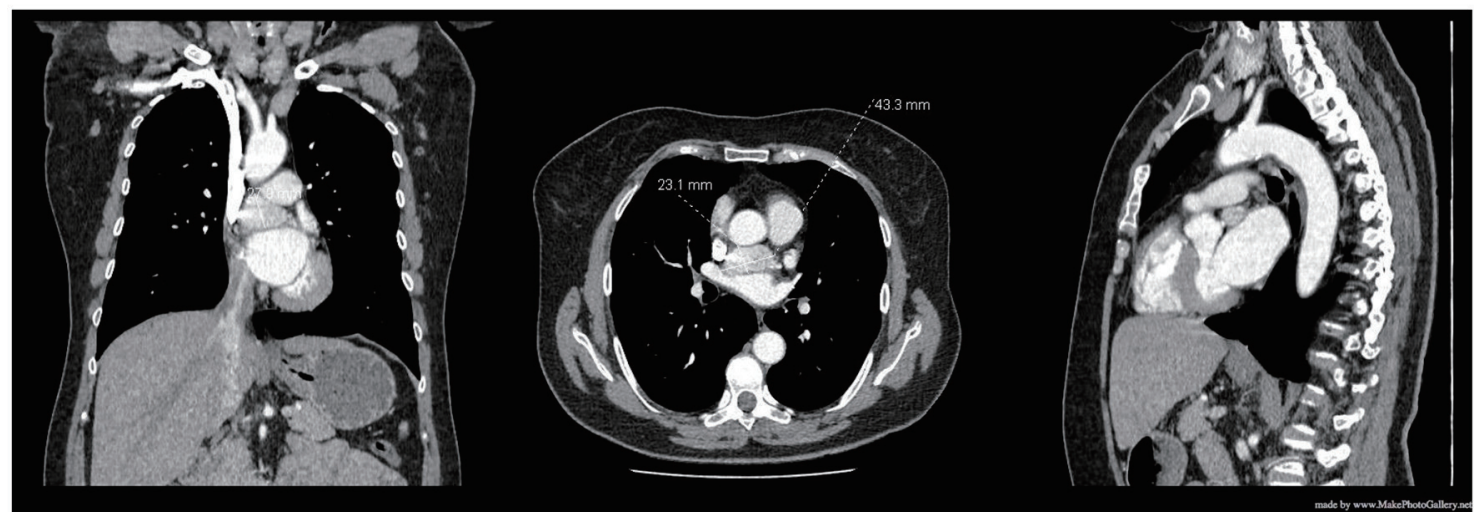

Figure 4. CT scan showing a mediastinal mass. CT: computed tomography.

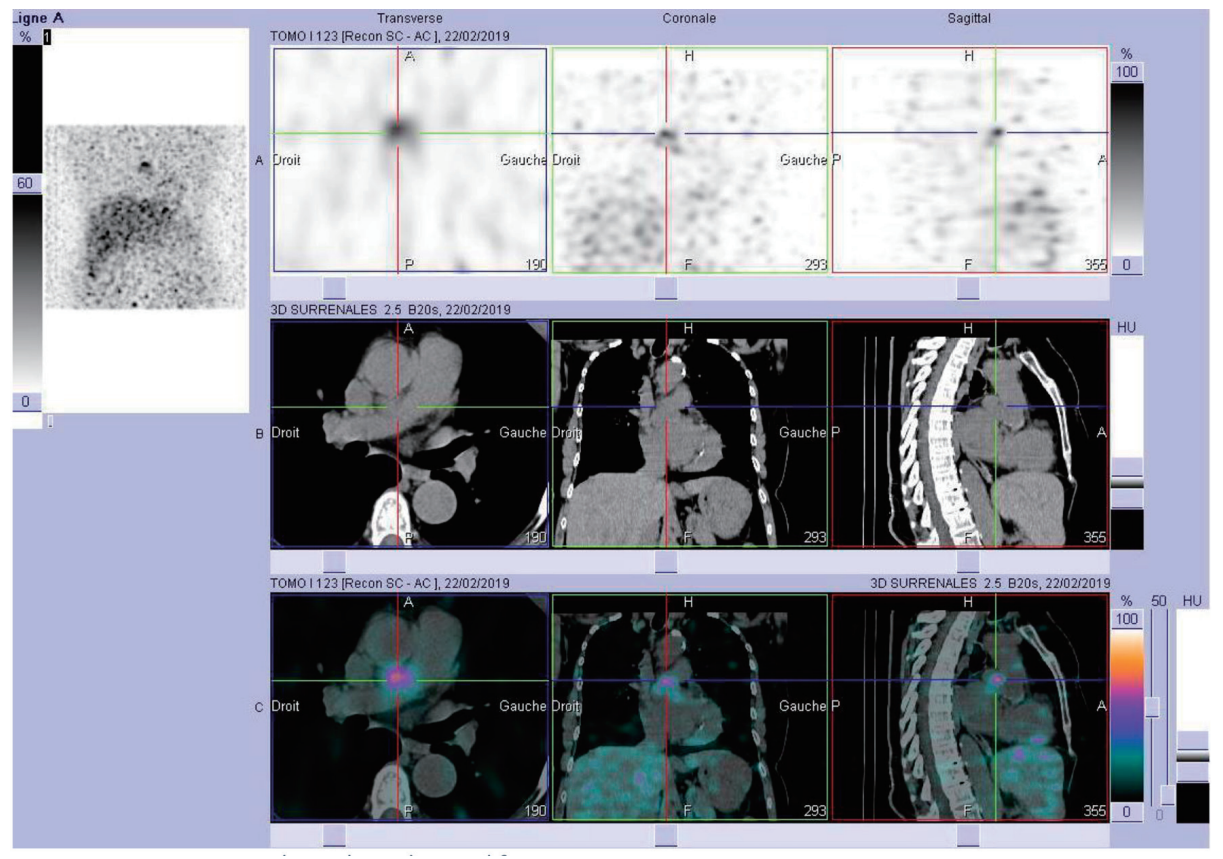

Figure 5. MIBG scintigraphy with mediastinal fixation. MIBG: metaiodobenzylguanidine. 

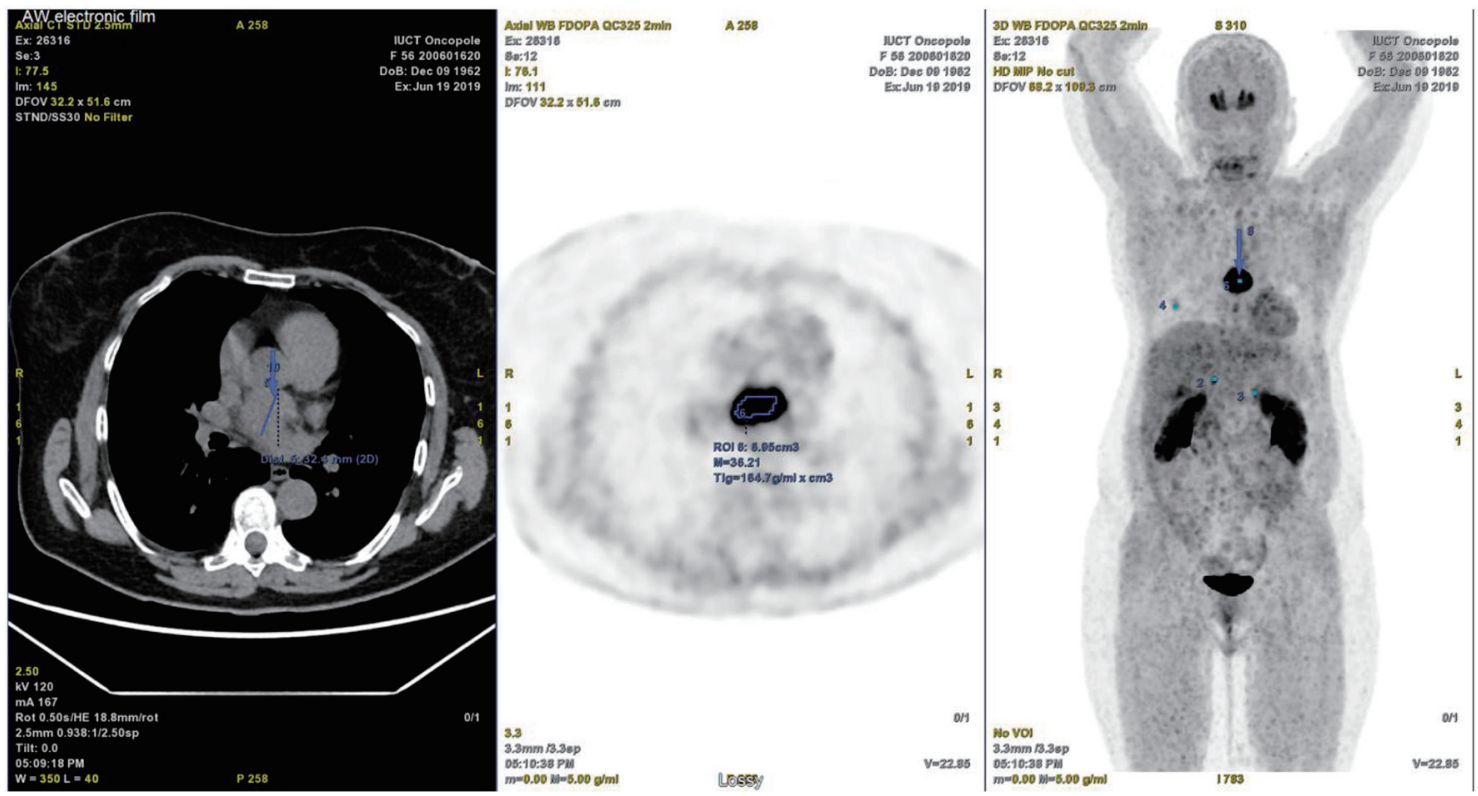

Figure 6. PET/CT scan with intense mediastinal fixation. PET/CT: positron emission tomography/computed tomography.

tension caused by fibromuscular dysplasia. PGL was diagnosed years later. We cannot confirm a causal association between the two entities; however, the management of fibro-dysplasia should take into consideration the presence of PGL which was not diagnosed early. Balloon angioplasty if indicated in the presence of a hidden PCC/PGL may be too dangerous.

In the literature, normal or borderline urinary catecholamines could be found in a patient with PCC [11]. For that, studies recommend undergoing MIBG in patients with normal biochemical testing when PCC is clinically suspected [12]. In our case, the patient did not present with early symptoms suggestive of PCC/PGL. However, earlier diagnosis of PGL would have been possible if we had considered this association especially in a patient presenting with recurrent RAS. Although this coexistence is rare, nevertheless making the diag- nosis of PGL in a fibromuscular dysplasia patient is crucial for an appropriate non-hazardous approach.

\section{Conclusion}

PGLs can present with nonspecific symptoms. The presence of resistant hypertension in a patient with significant or non-significant fibro-dysplastic lesion should evoke searching the coexistence of PGLs before managing patients with balloon angioplasty.

\section{Acknowledgments}

We acknowledge the authenticity of our work meeting all

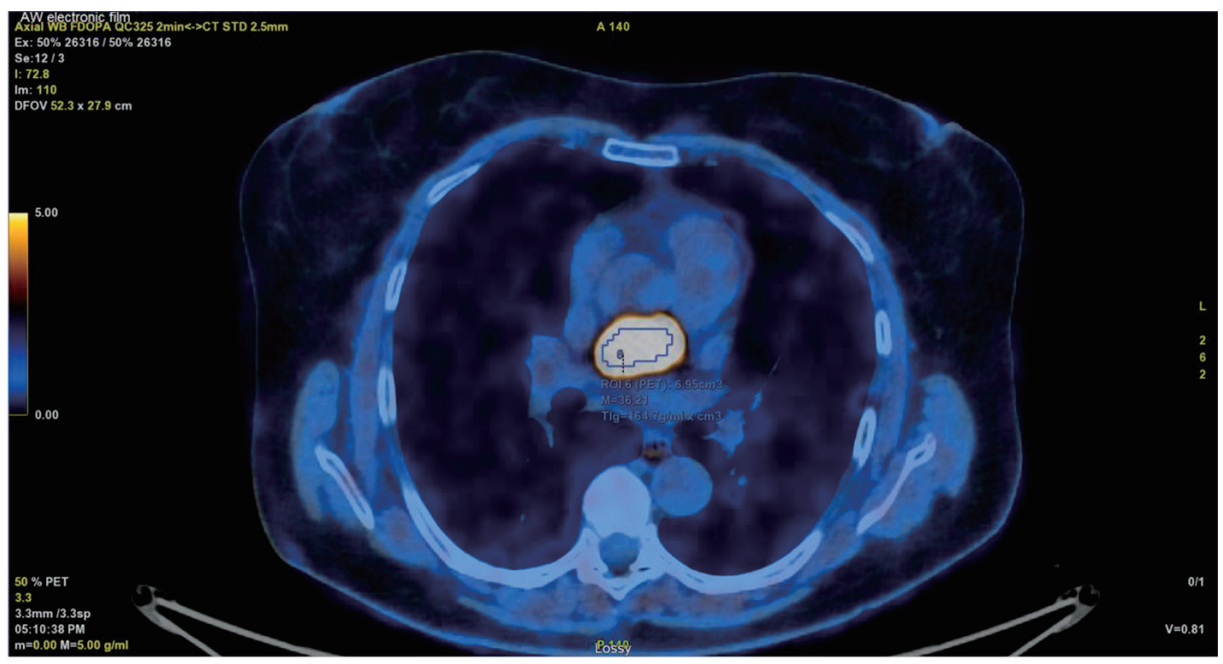

Figure 7. PET/CT with intense mediastinal fixation. PET/CT: positron emission tomography/computed tomography. 


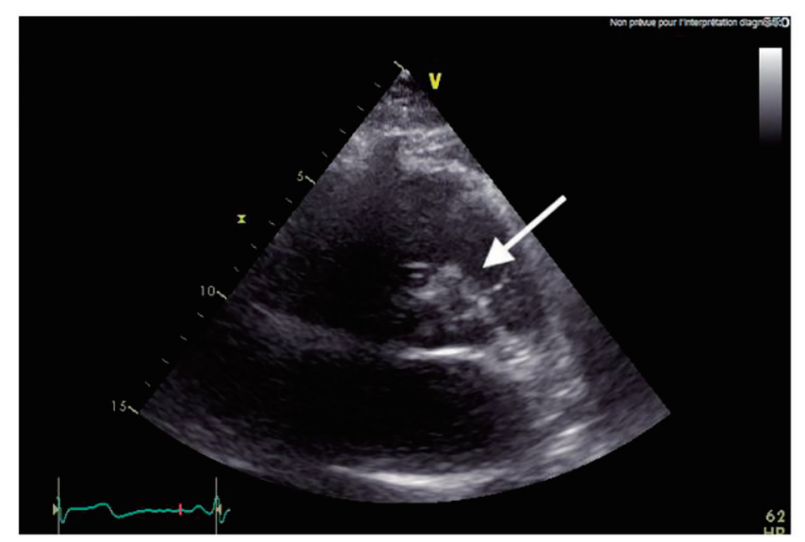

Figure 8. TTE with unusual mass. TTE: transthoracic echography.

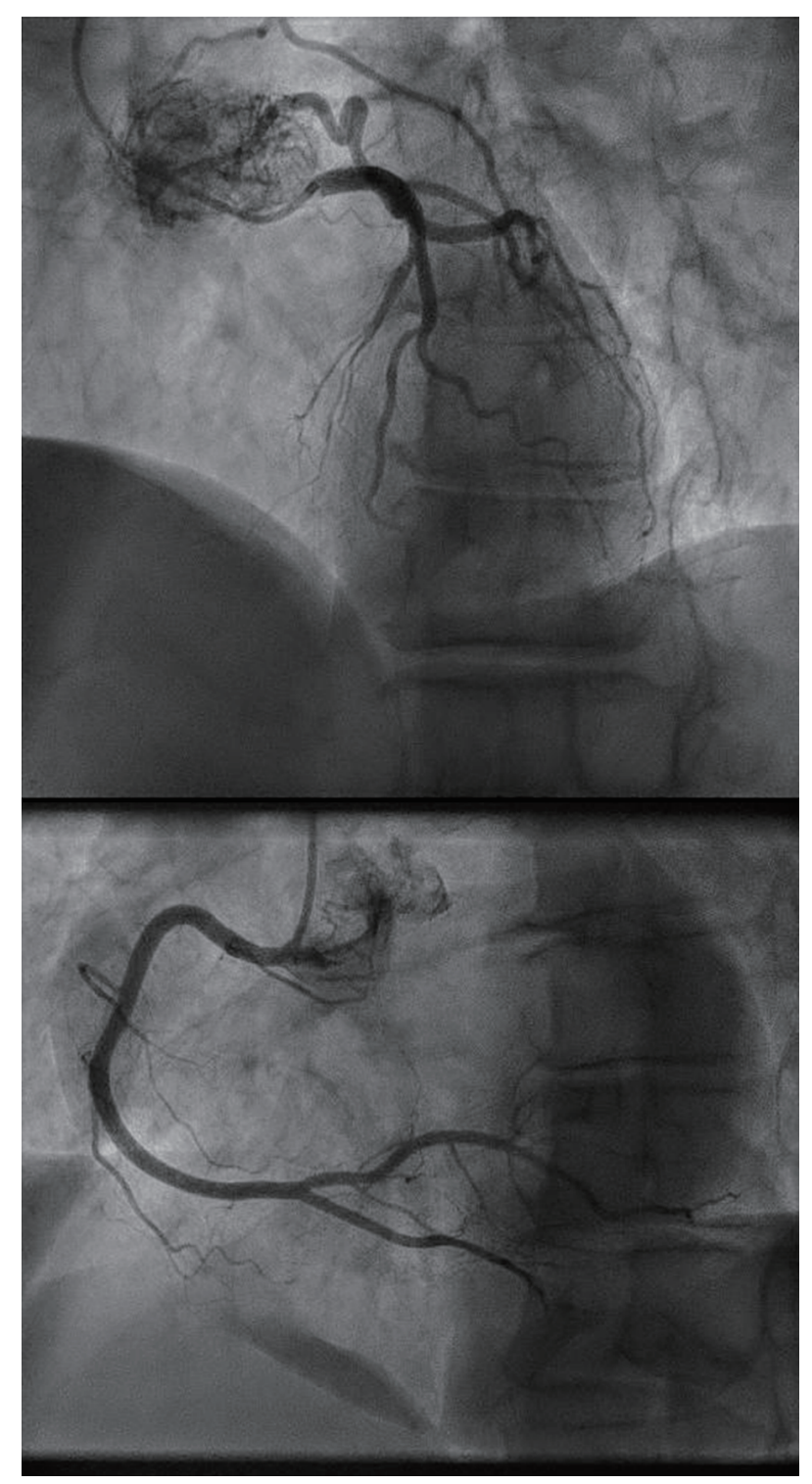

Figure 9. Vascularization of the mediastinal mass by two collaterals originating from the proximal and distal part of circumflex artery.

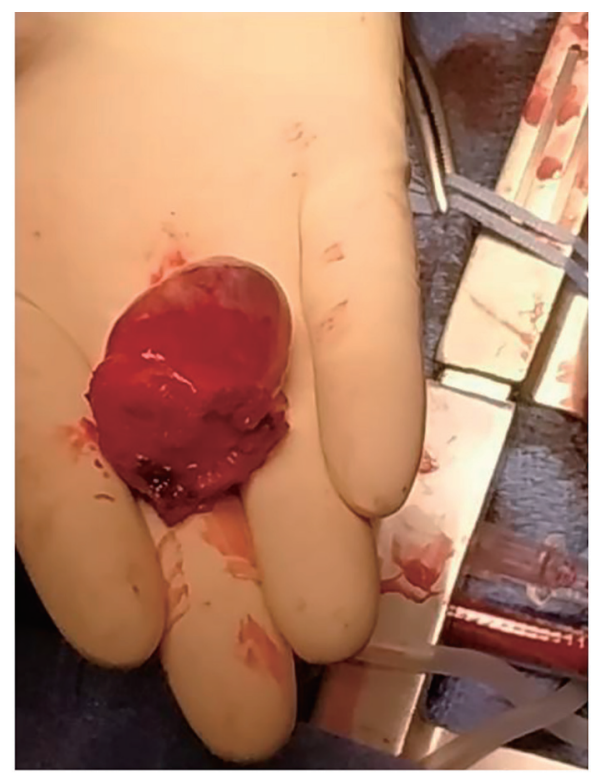

Figure 10. Total resection of the tumor.
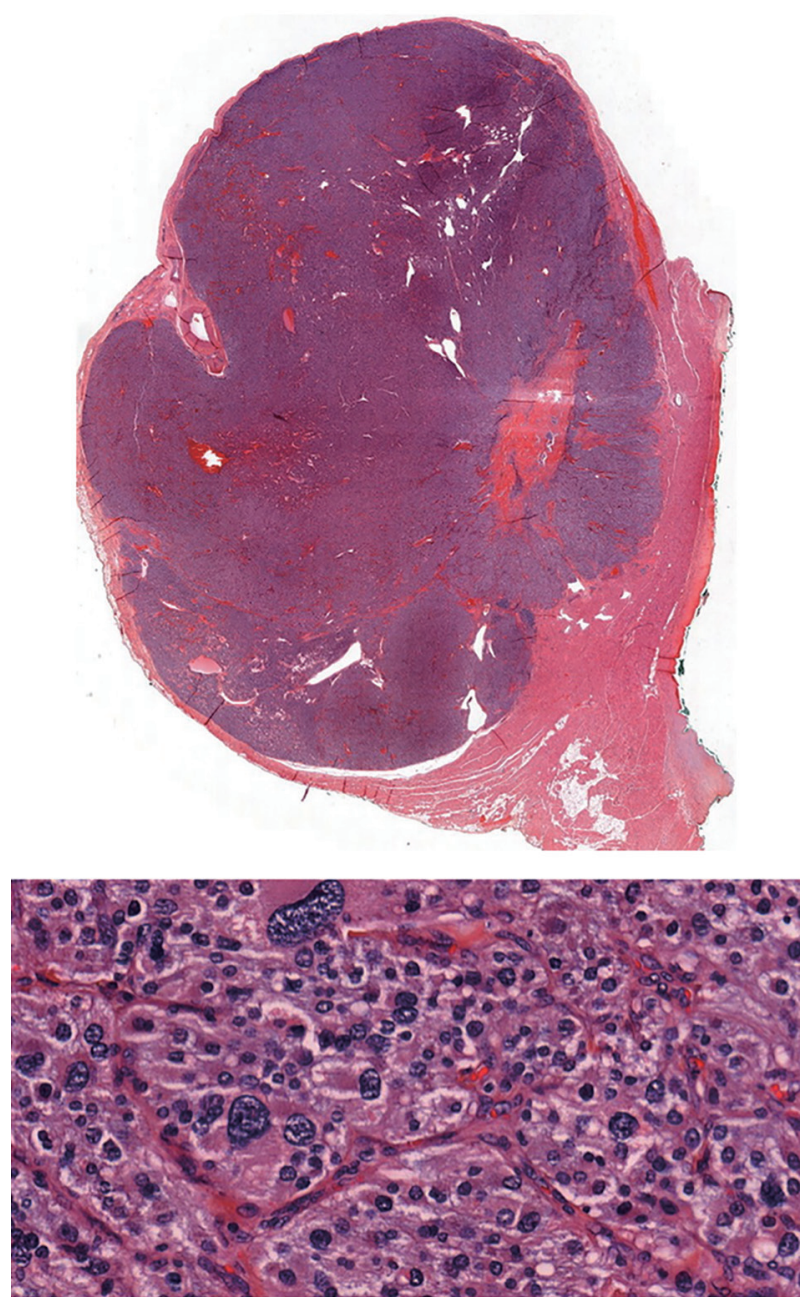

Figure 11. Histopathology specimen confirming the PGL. 
standards for publication.

\section{Financial Disclosure}

No funding source or grants.

\section{Conflict of Interest}

None to declare.

\section{Informed Consent}

Informed consent was obtained verbally from the patient.

\section{Author Contributions}

KE, RA and AM: study design, data collection, data interpretation, manuscript preparation, literature research and funds collection. MG: study design, data interpretation and manuscript preparation.

\section{References}

1. Rindi G, Klimstra DS, Abedi-Ardekani B, Asa SL, Bosman FT, Brambilla E, Busam KJ, et al. A common classification framework for neuroendocrine neoplasms: an International Agency for Research on Cancer (IARC) and World Health Organization (WHO) expert consensus proposal. Mod Pathol. 2018;31(12):1770-1786.

2. Chow LTC, Chan MHM, Wong SKC. Functional ulnar nerve paraganglioma: first documented occurrence in the extremity with hitherto undescribed associated ex- tensive glomus cell hyperplasia and tumorlet formation. Int J Surg Pathol. 2018;26(1):64-72.

3. Mandak JS, Benoit CH, Starkey RH, Nassef LA, Jr. Echocardiography in the evaluation of cardiac pheochromocytoma. Am Heart J. 1996;132(5):1063-1066.

4. Marchesi M, Biffoni M, Jaus MO, Nobili Benedetti R, Tromba L, Berni A, Campana FP. Surgical treatment of paragangliomas of the carotid body and other rare localisations. J Cardiovasc Surg (Torino). 1999;40(5):691-694.

5. Harrison JH, Gardner FH, Dammin GJ. A note on pheochromocytoma and renal hypertension. J Urol. 1958;79(2):173-178.

6. Gill IS, Meraney AM, Bravo EL, Novick AC. Pheochromocytoma coexisting with renal artery lesions. J Urol. 2000;164(2):296-301.

7. Sarathi V, Bandgar T, Lila AR, Deshpande AA, Dalvi AN, Patwardhan S, Shah NS. Coexistence of pheochromocytoma/praganglioma and renal artery stenosis. Indian J Endocrinol Metab. 2012;16(6):1009-1011.

8. Stanley JC, Gewertz BL, Bove EL, Sottiurai V, Fry WJ. Arterial fibrodysplasia. Histopathologic character and current etiologic concepts. Arch Surg. 1975;110(5):561566.

9. Brewster DC, Jensen SR, Novelline RA. Reversible renal artery stenosis associated with pheochromocytoma. JAMA. 1982;248(9):1094-1096.

10. Kishikawa H, Tsuji H, Takagi I, Yamakawa Y, Shimozato T, Honda K, Shibata A, et al. Hemorrhagic necrosis of pheochromocytoma associated with reversible renal artery stenosis. Jpn J Surg. 1986;16(1):46-51.

11. Zianni D, Tzanela M, Klimopoulos S, Thalassinos NC. Symptomatic pheochromocytoma with normal urinary catecholamine metabolites. Hormones (Athens). 2004;3(2):132-137.

12. Guller U, Turek J, Eubanks S, Delong ER, Oertli D, Feldman JM. Detecting pheochromocytoma: defining the most sensitive test. Ann Surg. 2006;243(1):102-107. 